\title{
SOLTEROS O CASADOS: ¿QUIÉN COME MEJOR? LA EVIDENCIA FAUNÍSTICA DEL ASENTAMIENTO MINERO HISTÓRICO DE CAPOTE, REGIÓN DE ATACAMA (CHILE)
}

\author{
Rafael Labarca, ${ }^{1}$ Elisa Calás ${ }^{2}$ y Francisco Rivera ${ }^{3}$
}

"Desde el punto de vista de sus múltiples usos, la vaca se divide en cierto número de trozos, de los que cada cual tiene distinta y especial aplicación".

(Morvan 1938:100).

\section{* Introducción}

Resumen

A partir de un análisis zooarqueológico se discute la posibilidad de detectar diferencias en las opciones dietarias de dos segmentos de la población, casados y solteros, del asentamiento minero histórico de Capote durante la primera mitad del s. XX. En base a la frecuencia de partes esqueletarias de taxones principalmente domésticos, así como a la abundancia y diversidad de éstos, se demuestra que el grupo casado se alimenta mejor que el grupo soltero, ya que el primero depende de las decisiones domésticas tomadas al interior de su núcleo familiar, mientras que el segundo se acoge a un régimen alimenticio minero tendiente a maximizar los recursos.

Palabras claves: zooarqueología - alimentación - sociedades complejas minería histórica.

\begin{abstract}
Based on zooarchaeological analyses, we discuss the possibility of detecting differences in the dietary choices of two population segments, singles and married, at the mining settlement of Capote during the first half of XX Century. Keeping in mind the frequency of skeletal parts of domestic taxa, and their diversity, it is demonstrated that the married are better fed than singles. The former depend on the decisions of their

women, while singles were subject to a that is primarily focused to a more restrictive diet that is primarily focused on resource maximization.
\end{abstract}

Key words: zooarchaeology - diet - complex societies - historic mining. Recibido: mayo 2008. Aceptado: mayo 2009.
El estudio de los huesos de animales provenientes de yacimientos arqueológicos históricos ha tenido un crecimiento sostenido desde la década de los 9o, en gran parte gracias al avance de la zooarqueología y de la arqueología histórica (Landon 2005). Debido a que su objeto de estudio se orienta fundamentalmente a entender el manejo de los recursos faunísticos en sociedades complejas de data relativamente reciente, las problemáticas de investigación y posibilidades interpretativas van mucho más allá de la simple documentación de la subsistencia dentro de un modelo de optimización (Gumerman 1997). La existencia de numerosas fuentes históricas de información, así como de materialidades asociadas, permite además contrastar, enriquecer y robustecer los resultados generados a partir de análisis exclusivamente faunísticos.

De acuerdo con Crabtree (1990), han sido tres las principales temáticas en las que se ha centrado la zooarqueología histórica: el rol de los animales en el intercambio y comercio, la identificación de distintos grupos étnicos, y la detección de diferencias sociales a partir de los desechos faunísticos. El presente trabajo se enmarca dentro de esta última línea de investigación. A partir de los restos faunísticos del yacimiento de Capote, un asentamiento minero utilizado durante

\footnotetext{
1 Universidad Nacional del Centro de la Provincia de Buenos Aires. Juan Moya Morales 910, Santiago, CHILE.Email: r.labarca.e@gmail.com

2 Parque Hornopirén Norte 8986, Santiago, CHILE.Email: elisa.calas@gmail.com

3 Elvira Garcés 1987, Depto.302, Santiago, CHILE. Email: franchurivera@gmail.com
} 
la primera mitad del s. XX, se caracterizan las opciones alimentarias de dos segmentos productivos de este enclave: casados y solteros. Se trata de dos grupos muy heterogéneos, conformados por individuos de distintas edades, provenientes de diferentes lugares geográficos, quizás de diversas etnias y extracciones sociales, los que sólo comparten condiciones laborales y de salario. Si bien a primera vista resulta difícil encontrar un común denominador que logre reunir ambos segmentos para un análisis, es justamente la comida, y particularmente el régimen alimentario al interior de Capote, uno de los elementos que permite su estudio comparativo, ya que los solteros se acogen al sistema de "pensión" compuesto por tres comidas diarias entregado en la cantina local, mientras que los casados se alimentan en sus propios hogares (Rivera et al. 2007). Teniendo como marco referencial el estudio de las diferencias socioeconómicas en zooarqueología histórica, complementado con información etnográfica y etnohistórica, discutimos cuál de estos dos grupos posee una dieta más variada y con mayor contenido cárneo, es decir, quién come mejor.

\section{$*$ Antecedentes}

\section{El estudio de la desigualdad social en la zooarqueología histórica}

En zooarqueología, el estudio de las diferencias sociales se basa en que los distintos grupos de clase tienen un acceso diferencial a los productos alimentarios (Crabtree 1990). Al encontrarse los consumidores inmersos en una economía de mercado, optarán por ciertos alimentos en desmedro de otros, de acuerdo con su poder adquisitivo e idealmente, teniendo en mente una relación costo-beneficio (Schulz y Gust 1983; Lyman 1987; Huelsbeck 1989, 1991). En términos metodológicos, para el análisis de este supuesto se ha considerado principalmente la frecuencia de partes esqueletarias, y la presencia o ausencia de ciertos taxones y su abundancia relativa (Crabtree 1990).

Respecto de la frecuencia de partes esqueletarias, tradicionalmente se ha establecido una relación directa entre unidad anatómica recuperada en el registro arqueológico (p.e., costilla) y corte de carne asociado a ésta definido históricamente (p.e., "asado de tira").. Debido a que los cortes de carne poseen distinto precio y rendimiento, se han elaborado diversos rankings en función de su valor comercial (p.e., Schulz y Gust 1983), complementados con la utilidad neta de cada corte (Lyman 1987; Huelsbeck 1989) y de cada unidad de consumo extraída de éste (Huelsbeck 1991). De esta manera, en un escenario ideal, aquellos consumidores con mayor poder adquisitivo optarán preferentemente por los cortes más caros, los que no necesariamente poseen el mayor rendimiento neto. Esta metodología, no obstante, solo puede ser aplicada en aquellos casos en donde existe una normativa clara y regularizada de los cortes de carne (Bowen 1998), generalmente ligada a un centro de producción y abastecimiento centralizado, donde los consumidores adquieren los productos ya procesados (Crabtree 1990). En contextos rurales, donde los grupos domésticos mantienen un pequeño número de ganado para su propio beneficio (Bowen 1998), o cuando se trata de carnicerías que poseen animales vivos (Schmitt y Zeier 1993), estas herramientas no arrojan resultados confiables.

Pero más complejo aún es sopesar el problema de aquellos cortes sin carne (Otto 1984, cit. en Landon 2005), ya que puede conducir a interpretaciones equivocadas de las opciones alimentarias de los grupos en estudio. En Chile es el Instituto Nacional de Normalización (INN) el encargado en la actualidad de definir y estandarizar los distintos cortes de vacuno y de otros animales domésticos para su venta. En su última edición (INN 1995), registra un total de 33 cortes de carne de bovino, de los cuales solo seis se venden con hueso adherido (18.18\%). Además, de una misma unidad anatómica se extraen hasta seis cortes (p.e., escápula), todos con precios diferentes, por lo que resulta imposible definir un ranking general utilizando una relación directa entre "corte-unidad ósea-precio". Esta situación no ha variado mayormente a través del tiempo: Pereira Salas (1977) lista 22 cortes, manteniendo las mismas seis piezas con hueso, mientras que en El Mercurio de Valparaíso de 1931, un listado de precios de carne indica la existencia de 25 cortes, en gran parte similares a los actuales, en los que se mantienen los cortes

\footnotetext{
4 Se trata de un corte comercial de vacuno, que se compone fundamentalmente de la porción medial de las costillas del animal y los músculos asociados. Se conoce también como "costilla derecha". En la Tabla 1 se detallan los cortes de vacuno y su composición ósea.
} 
con hueso (Tabla 1). Siguiendo el mismo procedimiento de Schulz y Gust (1983), Lyman (1987) y Huelsbeck (1989), al "rankear" los cortes de acuerdo a su precio, se puede advertir que los más caros son precisamente aquellos que no llevan hueso. De igual manera, en términos de costo-beneficio, los cortes más "rentables" no poseen unidad ósea adherida (ver Tabla 1). Esto quiere decir que cualquier estudio que discuta el estatus socioeconómico, o el poder adquisitivo de un determinado grupo teniendo como base exclusivamente un conjunto óseo de vacuno, se verá sesgado por la imposibilidad de detectar todos los cortes realmente adquiridos y consumidos. Aun cuando se estableciera una relación directa simple entre unidad ósea-corte-precio, se está obviando además, el hecho de que los distintos cortes se asocian a preparaciones culinarias particulares, las que en algunos casos son utilizadas transversalmente entre los consumidores, independiente del estatus socioeconómico o poder adquisitivo del grupo. De acuerdo con el Manual de Cocina de Lucía Larraín (1943: 5): "El primero de los caldos es, sin contradicción el caldo de vaca y constituye la parte más esencial de una buena sopa". La autora, indica que para un buen caldo, base para la preparación de una diversidad de recetas, se utiliza tanto carne (p.e., "pollo ganso"; ver Tabla 1), como huesos (p.e., hueso "chascón"). Esta situación indica que ciertas porciones de unidades óseas de las que se obtienen cortes sin hueso serían adquiridos después de su faenamiento en las carnicerías para cocinar sopas y caldos, y que por tanto su presencia en el registro arqueológico no sería indicativa de personas de estatus socioeconómico bajo. No obstante lo anterior, existe una relación entre la "sopa de huesos" y las clases menos acomodadas, como sugiere Morvan (1938) en Manual de Cocina: "Los huesos y las manos de las vacas suministran buena sustancia para caldos de servicio, abundantes en comunidades, colegios, etcétera" (Morvan 1938: 100).

Pese a las dificultades enunciadas anteriormente, otros animales domésticos pueden ayudar a discutir, de manera general, el tema de la frecuencia de partes esqueletarias y su relación con el poder adquisitivo. De acuerdo con el INN (2003) el faenamiento de los caprinos es mucho más sencillo que el de los vacunos ya que éste se divide en pierna (coxal, sacro, fémur, patella, tibia, fíbula, astrágalo, y tarsos), chuletas (vértebras lumbares, vérte-

\footnotetext{
5 Fémur proximal.
}

bras torácicas y porción dorsal de las costillas), costillar (costillas y esternón) y miembro anterior (vértebras cervicales, vértebras cervicales hasta la quinta, escápula, húmero, radio, ulna, carpos). Todos los cortes llevan hueso y el faenamiento final está a cargo del consumidor. Este modo de procesamiento no ha variado mayormente con el tiempo ya que la lista de precios y cortes publicada en El Mercurio de Valparaíso de 1931 indica que las piernas y chuletas costaban $\$ 3.50$ el kilo, mientras que la espaldilla, costillas y espinazo $\$ 3.10$. Los manuales de cocina ${ }^{6}$ de la época consultados (Morvan 1938; Gracia 1942; Larraín 1943), confirman esta impresión ya que los platos descritos coinciden con las unidades de faenamiento publicadas en el periódico mencionado.

La segunda metodología utilizada para discutir desigualdades sociales es la presencia de determinados taxones y su frecuencia comparativa. Así, animales de precio o connotación social inferior deberían encontrarse mayormente representados en contextos de clases sociales más bajas. En contrapartida, la presencia de ciertas especies exóticas o difíciles de conseguir atendiendo la situación geográfica del yacimiento, indicaría la presencia de clases más acomodadas (Reitz 1986b; Crabtree 1990; Crader 1990; Landon 2005). Se debe tener presente que un producto puede cambiar su carácter "exclusivo", y por tanto su precio, de un lugar a otro dependiendo de su disponibilidad en el medio local (Huelsbeck 1991; Schmitt y Zier 1993). El ejemplo más claro es la variación en la abundancia y en el precio de los mariscos y pescados entre la costa y el interior. Teniendo en consideración la lista de precios publicada en El Mercurio de 1931, el cordero pareciera ser más caro que el vacuno; no obstante, se debe tener presente que los precios son publicados en Valparaíso (Chile Central) donde tradicionalmente la crianza de vacunos es más popular que la de ovinos. Basta ver los valores publicados en el diario La Reforma de Calama de 1932 ( $\mathrm{n}^{\circ}$ 14, año 1 ) donde se menciona que el kilo de carne de vacuno con hueso costaba $\$ 2.80$, y el kilo de carne sin hueso $\$ 3.20$; el cordero, por su parte tenía un valor de $\$ 2.40$ el kilo de chuletas y pierna, y $\$ 2.20$ el kilo de cazuela (costillas).

\footnotetext{
${ }^{6}$ A juzgar por la descripción de los cortes y los comentarios del INN (2003) la espaldilla correspondería a las primeras vértebras torácicas en conjunto con la escápula, y el espinazo haría referencia a las vértebras cervicales.
} 


\begin{tabular}{|c|c|c|c|c|c|c|}
\hline Cortes (1) & $\begin{array}{l}\text { Presencia / } \\
\text { ausencia de } \\
\text { Hueso }\end{array}$ & Unidades óseas relacionadas (2) & $\begin{array}{l}\text { Precio (pesos/ } \\
\text { kilo) (3) }\end{array}$ & $\begin{array}{l}\text { Ranking } \\
\text { precios }\end{array}$ & $\begin{array}{l}\text { Peso corte } \\
\text { completo } \\
(\mathbf{k})(4)\end{array}$ & $\begin{array}{c}\text { Ranking } \\
\text { costo/ } \\
\text { beneficio* }\end{array}$ \\
\hline Filete & A & $\begin{array}{l}\text { Cuerpo vertebral de vértebras torácicas 11-13, segmentos } \\
\text { dorsales de costillas correspondientes, vértebras } \\
\text { lumbares, cuerpo del ilion y epífisis proximal del fémur }\end{array}$ & 4.4 & 1 & 3 & 9 \\
\hline Lomo liso & A & $\begin{array}{c}\text { Cuatro últimas vértebras torácicas, segmentos dorsales } \\
\text { de costillas 10-13, vértebras lumbares hasta procesos } \\
\text { espinosos, cresta ilíaca }\end{array}$ & 3.5 & 2 & 5 & 6 \\
\hline Lomo vetado & A & $\begin{array}{c}4^{\mathrm{a}} \text { a } 9^{\mathrm{a}} \text { vértebras torácicas, segmentos dorsales de } \\
\text { costillas respectivas }\end{array}$ & 3.5 & 2 & 5 & 6 \\
\hline Asiento picana & A & Coxal, fémur & 3.5 & 2 & 3 & 6 \\
\hline Punta de ganso & A & Sacro, coxal, fémur, tibia proximal, calcáneo & 3.5 & 2 & 3 & 6 \\
\hline Posta negra & A & Isquion, pubis, fémur, tibia & 2.6 & 3 & 7 & 4 \\
\hline Posta rosada & A & Cuerpo del coxal, fémur, tibia & 2.6 & 3 & 5 & 4 \\
\hline Pollo ganso & A & Isquion, fémur, calcáneo & 2.6 & 3 & 4 & 4 \\
\hline Pollo de barriga & A & Vértebras lumbares & 2.6 & 3 & 1 & 4 \\
\hline Ganso & A & Sacro, coxal, fémur, tibia proximal, calcáneo & 2.6 & 3 & 3 & 4 \\
\hline Horqueta & A & Sin datos & 2.6 & 3 & Sin datos & 4 \\
\hline Plateada & A & Costillas, húmero proximal, escápula & 2.3 & 4 & 3 & 3 \\
\hline Sobrecostilla & A & Costillas, vértebras torácicas, escápula & 2.3 & 4 & 5 & 3 \\
\hline Malaya & A & Costillas & 2.3 & 4 & 2.5 & 3 \\
\hline Guachalomo & A & Vértebras cervicales, vértebras lumbares, escápula & 2.3 & 4 & 4 & 3 \\
\hline Tapapecho & A & Costillas, esternón, húmero & 1.9 & 5 & 5 & 2 \\
\hline Manotilla & A & Sin datos & 1.9 & 5 & Sin datos & 2 \\
\hline Estomaguillo & A & Segmento ventral de las costillas & 1.9 & 5 & 2 & 2 \\
\hline Tapaposta & A & Sin datos & 1.9 & 5 & Sin datos & 2 \\
\hline Costilla derecha & P & Segmento medial de las costillas & 1.9 & 5 & 4 & 7 \\
\hline Costilla coluda & $\mathrm{P}$ & Segmento ventral de las costillas & 1.9 & 5 & 4 & 7 \\
\hline Costillas arqueadas & $\mathrm{P}$ & Segmento dorsal de las costillas & 1.05 & 6 & 3 & 5 \\
\hline Barranquilla & $\mathrm{P}$ & Vértebras lumbares & 1.05 & 6 & Sin datos & 8 \\
\hline Huesos de pierna & $\mathrm{P}$ & Fémur distal, patella, tibia, astrágalo, calcáneo, tarsos & 1.05 & 6 & 6 & 8 \\
\hline Huesos de mano & $\mathrm{P}$ & Húmero distal, radioulna, carpos & 1.05 & 6 & 4 & 8 \\
\hline Cogote & A & Vértebras cervicales, húmero, esternón, escápula & 0.45 & 7 & 4 & 1 \\
\hline
\end{tabular}

Tabla 1. Resumen de cortes de carnes y precios de vacuno en Chile en 1931. (1) De acuerdo con la lista publicada en El Mercurio de Valparaíso $n^{\circ}$ 34204, junio 17 de 1931; (2) De acuerdo con el INN (1995) y Amtmann (1967); (3) De acuerdo con la lista publicada en El Mercurio de Valparaíso $n^{\circ}$ 34204, junio 17 de 1931; (4) De acuerdo con Amtmann (1967). * El peso neto de los cortes con hueso se calculó considerando $50 \%$ para la costilla derecha y coluda y $30 \%$ para los huesos de la pierna y mano. 


\section{El yacimiento de Capote, la dinámica en los asentamientos mineros y sus implicancias para entender el registro faunístico}

El mineral aurífero de Capote se ubica en la región de Atacama en el norte de Chile, a unos $740 \mathrm{~km}$ al norte de Santiago (Figura 1). Si bien fue "descubierto" a principios del s. XVIII, aparentemente habría sido explotado desde tiempos prehispánicos aunque no existen evidencias de ello (Rivera 2008). Durante la Colonia se habría iniciado la explotación de los primeros piques mineros sin generar asentamientos permanentes (Rivera et al. 2007). Durante el s. XIX y comienzos del XX, el mineral de Capote pasó por diferentes dueños quienes abrieron nuevos socavones, pero de manera relativamente informal y con moderado éxito. Es recién en 1936 cuando se registra el momento de mayor auge en la explotación de Capote con el empresario Paulino Callejas y la Sociedad Capote Aurífero de Freirina, ya que comienza la explotación sistemática e industrial del mineral. Se construye un asentamiento permanente destinado a la administración, procesamiento del mineral y vivienda de los trabajadores. Durante su mayor apogeo contó con farmacia, pulpería, panadería, plaza, teatro, dos escuelas, imprenta y canchas de fútbol, todas comodidades para los cerca de 800 obreros, 60 empleados y sus respectivas familias (Rivera et al. 2007). Hacia 1950 comienzan lentamente a paralizarse las obras producto del agotamiento del mineral. En 1959 la mina se cierra definitivamente y el campamento es abandonado.

La vida en Capote giraba en torno al mineral, el que se extraía en gran medida a través de técnicas manuales por obreros que residían en el asentamiento central. Para tales efectos, la Sociedad Capote Aurífero de Freirina construyó distintas instalaciones residenciales en un área delimitada, dividida en un sector de obreros solteros y otro de casados (ver Figura 1). Así recuerda Juan Varela, trabajador de la época:

"Mire, Capote tenía hecho unos camarotes, unos camarotes largos, más o menos unas 10 piezas para los solteros y los casados tenían casas, de dos piezas con una cocina, un comedor, casas de las muy corrientes que hay en las minas" (Rivera et al. 2007: 92).
Tanto el salario que recibían, como el trabajo que realizaban no diferían mayormente entre solteros y casados, por lo que no es posible hablar de dos clases sociales distintas. La mayor diferencia recaía en los lugares de habitación y en el régimen alimentario. En los casados, era la mujer quien se encargaba de las labores domésticas y la preparación de los alimentos, mientras que los solteros se incorporaban al régimen de "pensión", el que incluía tres comidas diarias por un monto mensual fijo. Éstas eran preparadas en la cantina por mujeres que se dedicaban exclusivamente a esta actividad, debido a la gran cantidad de comida que se cocinaba diariamente. Laura Rodríguez, quien trabajó en este lugar, relata muy bien el extenuante trabajo:

"Cuando teníamos pensionistas, me levantaba a las cuatro de la mañana, porque había que hacer el pan, había que dejar las papas peladas en la noche porque no se pelaba un kilo, ni dos... un saco, medio saco de papas porque eran 80 pensionistas, había que hacer el pan, atender la gente desde las seis de la mañana [...], hasta en la noche y en la noche como a las 11" (Rivera et al. 2007: 92).

En general, y como es característico en todos los asentamientos mineros norteños emplazados en ambientes desérticos, su funcionamiento se encontraba completamente supeditado a envíos externos. Desde el agua, que subía diariamente en camiones aljibe, hasta los artículos de consumo diario provenían de los poblados más cercanos, como Huasco, Freirina o Vallenar (Rivera et al. 2007). Al interior del asentamiento, era la pulpería el principal centro de abastecimiento de provisiones, cuyos precios eran controlados por la administración. Sin embargo, no todos los alimentos que consumían los capotinos eran adquiridos en este almacén, ya que tres o cuatro veces por semana subían al mineral de Capote comerciantes ambulantes desde los pueblos cercanos. En este sentido, es importante mencionar que, contrario a lo registrado en las oficinas salitreras emplazadas más al norte (González 2002), en Capote no se ocuparon fichas de salario, y los trabajadores recibían su sueldo en dinero, lo que facilitó el acceso a los productos que ofrecían los afuerinos (Rivera et al. 2007). La presencia de vendedores ajenos a la sociedad explotadora se habría traducido en una suerte de competencia entre éstos y la pulpería, lo que sin duda ayudó a regular los precios de mejor ma- 


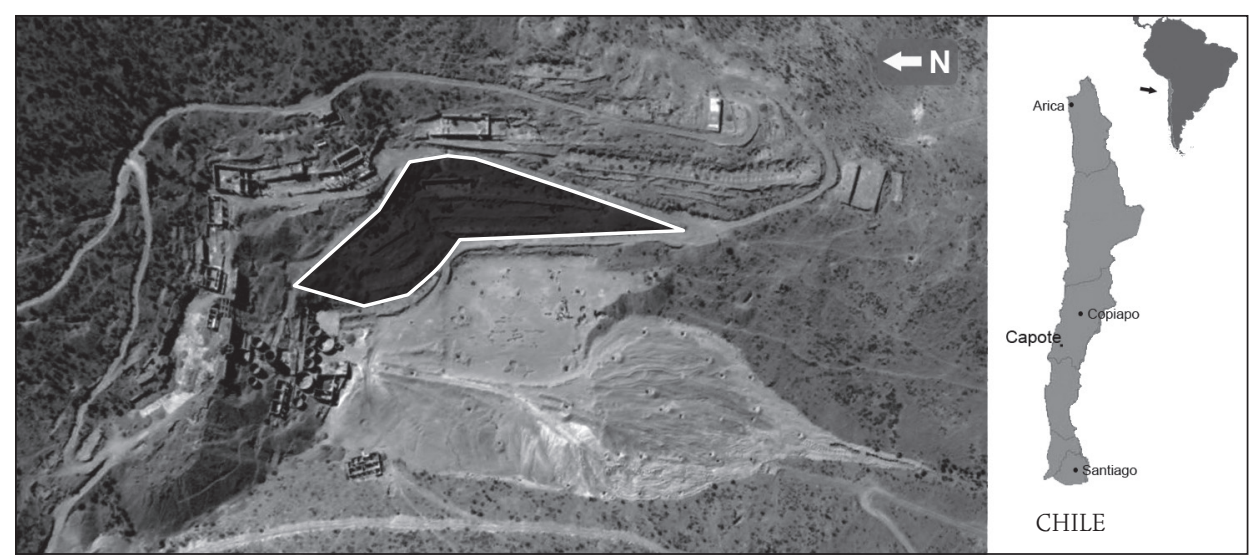

Figura 1. Ubicación y vista aérea del yacimiento de Capote. El polígono de líneas blancas al centro marca el área habitacional.

nera, homologándolos al mercado regional. Don Alirio, "pioneta"7 de un camión de transportes, recuerda una parte de los productos que se comercializaban:

“...lo que nosotros llevábamos eran frutas, melones, sandías, cosas que aquí también se cosechan; peras, brevas, higos, frutillas, la aceituna; verduras como la betarraga, lechuga, zapallo, también abarrotes como: arroz, fideos, legumbres, harina, azúcar, poroto, la carne de vacuno, cabrito y gallina de corral, salmón, mortadela, paté [...] todo lo que llevaba la gente lo vendían, no sobraba nada" (Rivera et al. 2007: 86).

No es mucha la información que existe sobre el menú de los capotinos, pero en principio, éste no difería mayormente entre casados y solteros: se comía mucho arroz, legumbres, papas, huevos y carne, incluso en el desayuno. Las preparaciones incluían cazuela y porciones de carne asada (Rivera et al. 2007). De acuerdo con las entrevistas recopiladas, la carne consumida era principalmente de vacuno, cabra y aves de corral.

Diversas expectativas pueden surgir de los párrafos precedentes. En primer término en el campamento de Capote los productos cárneos eran adquiridos en puestos de venta, lo que permite suponer que los capotinos no habrían criado animales domésticos para el consumo personal en las áreas residenciales. Esto es parti-

\footnotetext{
7 "Pioneta" en la jerga popular; Peoneta: "Auxiliar de vehículos de carga" (DICRAE); cargador.
}

cularmente válido para los solteros, quienes dependían íntegramente de los menús ofrecidos en la cantina. Respecto de los casados, podemos suponer una situación relativamente similar, aunque no se descarta que las mujeres hayan criado animales pequeños que requieren de cuidados menores, como gallinas. Debido a que tanto las administradoras de las cantinas como las esposas de los obreros compraban los productos en los mismos lugares, podemos suponer que, en principio, el menú entre solteros y casados habría sido relativamente similar, es decir, los mismos taxones (cabrito y vacuno) y las mismas preparaciones. No obstante, es posible sugerir eventuales diferencias a ser evaluadas en el registro arqueológico. La primera es la calidad de los cortes, particularmente en el caso del vacuno. Producto de que la cantina debía alimentar diariamente a un número importante de obreros, es factible pensar que ésta habría tendido a minimizar los costos, y por tanto, sus preparaciones habrían sido realizadas con cortes más baratos, o quizás un mayor número de preparaciones líquidas, lo que se traduciría en un número comparativamente más elevado de cortes de vacuno apropiados para este plato. De manera opuesta, si es que los casados se alimentaron de mejor manera, habrían accedido a cortes de vacuno sin hueso, y por lo tanto, su registro sería comparativamente más exiguo. En relación al cabrito, debido a su mayor disponibilidad regional tanto en la actualidad como en tiempos históricos (p.e., Pizarro y colaboradores. 2006), es posible que se encuentre de manera abundante en los dos 
contextos, pero quizás con unidades de mayor costo en las habitaciones de los casados. Por último, se puede esperar una mayor diversidad de taxones entre los casados, debido a las variaciones individuales en la preparación de platos en este segmento. En la cantina, en cambio, es esperable un menú mucho más homogéneo.

Por otra parte, debido a que el campamento no producía los bienes que demandaba, es factible suponer que los recursos cárneos habrían llegado procesados al asentamiento, ya sea en forma de cortes listos para su venta, o bien, unidades de faenamiento de nivel mayorista, los que habrían sido reducidos en el asentamiento minero. En términos del registro óseo resulta interesante discutir la presencia de dos puntos de venta de productos cárneos: uno "oficial" como es la pulpería, y otro informal, como son las ferias compuestas por los vendedores ambulantes. Aquí nos enfrentamos a un problema de difícil solución arqueológica ya que en teoría se dan cita dos modelos distintos: por un lado, los productos faenados de la pulpería que representan el modelo "urbano", asociado a mayor estandarización en los cortes así como a rangos etáreos acotados de los individuos (Bowen 1998); y por otro, el modelo "rural", que de manera opuesta, se traduciría en la venta de cortes fuera de norma (Reitz 1986a; Landon 1997; Bowen 1998). Idealmente, la frecuencia comparativa de estos dos modelos podría indicar la preferencia de un sistema de abastecimiento sobre otro entre los consumidores de Capote.

\section{* Material y métodos}

A partir de las fichas de arquitectura, las excavaciones arqueológicas y la información etnográfica, el asentamiento de Capote se habría dividido en cuatro grandes sectores: área de trabajo, área habitacional, área de espacios públicos y área de basurales. Se realizó un total de 15 pozos de sondeo de 1 x $0.5 \mathrm{~m}$, excavados con niveles artificiales de $10 \mathrm{~cm}$, distribuidos representativamente entre estos sectores (Rivera et al. 2007). Los restos óseos recuperados ascienden a 1023 especímenes, provenientes en su mayoría del área habitacional ( $\mathrm{n}=808 ; 78.98 \%$ del total) (Tabla 2 ). En este último sector se centra el presente trabajo, ya que es aquí donde es posible discutir la variación en los hábitos alimenticios entre casados y solteros. En el área habi- tacional se excavó un total de siete pozos de sondeo, tres en el sector de solteros y cuatro en el sector de casados. ${ }^{8}$

El análisis se llevó a cabo con todos los especímenes identificables, es decir, todas aquellas unidades óseas que presentaran porciones articulares, agujeros nutricios u otro accidente anatómico que permitiese su adscripción a una unidad anatómica en particular, y por tanto, a un grupo taxonómico definido. En esta etapa del análisis se utilizaron criterios osteomorfológicos a partir de esqueletos de referencia depositados en la Facultad de Ciencias Veterinarias y Pecuarias de la Universidad de Chile y guías osteológicas de fauna introducida (Boessneck et al. 1964; Boessneck 1980, Prummel y Frisch 1986; Adaro y Olivares 2006). La resolución en la asignación taxonómica de este segmento alcanzó distintos niveles de precisión debido a que no todas las unidades anatómicas revisadas fueron igualmente diagnósticas.

Las medidas de cuantificación utilizadas varían levemente en relación a las tradicionalmente empleadas en zooarqueología, puesto que diversos trabajos han discutido la validez de unidades como el MNI al analizar restos óseos de sitios arqueológicos históricos (Lyman 1987; Huelsbeck 1989). Esto se basa en el hecho de que los consumidores históricos no acceden a las carcasas completas, sino que a porciones de ellas, en función de las preferencias alimentarias, tipo de plato a cocinar, posición social, entre otros (Huelsbeck 1991). Lyman (1987) y Huelsbeck (1991) sugieren utilizar los conceptos de "unidad de faenamiento" y "unidad de consumo". El primero hace referencia al segmento mayor faenado del cual se extraen las unidades de consumo. Por ejemplo, en la actualidad, de una unidad de faenamiento como el "asado de tira" se extraen 12 unidades de consumo (UC), las que corresponden a las porciones de costillas que finalmente son utilizadas. De esta manera, se optó por emplear una metodología mixta que incluyó el NISP, es decir, el número de especímenes óseos identificados en un conjunto óseo por taxón (Grayson 1984) y la unidad de consumo, que se refiere a todo aquel fragmento de resto óseo que conforma una unidad potencialmente consumible (Lyman 1987; Huelsbeck 1991). En este análisis, una unidad de consumo se identifica como tal

\footnotetext{
8 Datos generales respecto a resultados de las otras áreas intervenidas se pueden encontrar en Labarca y Calás (2007 Ms).
} 


\begin{tabular}{|l|c|c|c|c|c|}
\hline Restos analizados & $\begin{array}{c}\text { Área de } \\
\text { trabajo }\end{array}$ & $\begin{array}{c}\text { Área } \\
\text { habitacional }\end{array}$ & $\begin{array}{c}\text { Área de espacios } \\
\text { públicos }\end{array}$ & $\begin{array}{c}\text { Área de } \\
\text { basurales }\end{array}$ & Total \\
\hline Hueso plano & 3 & 130 & 21 & 23 & 177 \\
\hline Fgto. mínimo & 7 & 266 & 21 & 15 & 309 \\
\hline Fgtos. diáfisis & 10 & 196 & 12 & 17 & 235 \\
\hline Astillas & 2 & 15 & 4 & 3 & 24 \\
\hline Identificados & 8 & 201 & 5 & 64 & 278 \\
\hline Total & 30 & 808 & 63 & 122 & 1023 \\
\hline
\end{tabular}

Tabla 2. Resumen de restos óseos analizados.

al poseer por lo menos una huella de aserrado que indique la reducción del elemento anatómico original en una porción menor. Finalmente, la nomenclatura de los cortes de vacuno se encuentra resumida en la Tabla 1.

Para controlar la incidencia de distintos agentes naturales en la preservación del conjunto, se consideraron los siguientes atributos tafonómicos: meteorización (analizada utilizando la escala propuesta por Behrensmeyer [1978]); marcas de abrasión producidas por partículas sedimentarias (Shipman 1981); marcas por pisoteo y arrastre (Behrensmeyer 1982; Behrensmeyer et al. 1986); marcas producidas por carnívoros durante el consumo de restos óseos (Binford 1981; Haynes 1983) y acción de roedores (Lyman 1994). Estas categorías sólo fueron consignadas para los especímenes identificados.

En relación con las modificaciones de carácter antrópico presentes en la muestra, se distinguieron cuatro tipos: marcas de corte, raspado (Binford 1981; Mengoni 1988, 1999), corte con sierra9 y fracturas traumáticas por percusión (Johnson 1983), reflejadas por negativos de impacto, derivados de fractura y lascas óseas. Finalmente se consideraron las huellas de exposición al fuego (Lyman 1994) en una escala medida por la coloración de los restos (exposición moderada: marrón; quemado: negro; calcinado: blanco). Estas categorías sólo fueron consignadas para los especímenes identificados. Todas aquellas unidades con huellas de sierra, fueron descritas y dibujadas siguiendo a Lyman (1977).

\footnotetext{
${ }^{9}$ Se diferencia de una huella de corte, porque el objetivo de su realización es cortar el hueso, es decir generar unidades de faenamiento o consumo, a diferencia de las huellas de corte tradicionales las que tienen por objeto cortar tejidos blandos (Lyman 1977, 1994).
}

\section{* Resultados y discusión}

Diversos autores han puesto en evidencia la necesidad de cotejar los procesos postdepositacionales que puedan afectar a los restos faunísticos históricos (p.e., Landon 2005). En este sentido, el área habitacional de Capote presenta en general una buena conservación, a excepción de la meteorización, la que se registra en cerca de $18 \%$ de la muestra, aun cuando el Estadio 1 es el más popular. Las huellas de otros agentes naturales como roedores, abrasión y pisoteo se encuentran prácticamente ausentes. Sólo las marcas de carnívoros y raíces exhiben un número comparativamente mayor (aproximadamente 7\%), pero aún así no afectaron al conjunto de manera significativa.

De acuerdo con lo argumentado, la presencia de ciertos taxones y la frecuencia relativa de éstos podrían ayudar a discutir los hábitos alimentarios observados entre los obreros solteros y casados en Capote. La Figura 2, grafica el porcentaje de NISP de los distintos taxones identificados en el área habitacional (NISP identificados casados: 123; NISP identificados solteros: 73). En concordancia con lo recopilado etnográficamente, se encuentran representados vacuno (Bos taurus), caprino, el que de acuerdo con las guías de referencia debería corresponder en su gran mayoría a cabra (Capra hircus), cerdo (Sus scrofa), un ave grande no determinada (posiblemente pato o gallina) y restos de peces no identificados. Entre estos dos segmentos de la población, el vacuno y los caprinos son las especies más consumidas y dentro de éstas, la segunda es la más utilizada. Esta situación coincide con su mayor disponibilidad en la región, lo que hace suponer que sus cortes eran más abundantes tanto en la pulpería como en la feria, y por tanto, sus precios eran inferiores. Al compa- 
rar las frecuencias de los taxones resalta el hecho de que los solteros aparentemente consumen más vacuno que los casados, mientras que los casados hacen lo propio con el caprino. Esta situación, en principio indicaría que los solteros accederían de manera más regular a productos más caros y de menor disponibilidad. No obstante, debemos tener presente la frecuencia de partes esqueletarias, punto sobre el que volveremos más adelante. Fuera de los dos taxones mayores, se observa que otras especies se encuentran prácticamente ausentes en el menú de los solteros a excepción de las aves, las que sin embargo tienen una muy baja representación. Los casados por su parte, poseen una dieta más variada, ya que se habrían alimentado, además, de cerdo y peces, estos últimos quizás con un precio mayor debido al emplazamiento del sitio. En relación con esto, Juan Videla, obrero de Capote menciona: "Pescado había, como queda cerca de Carrizal [caleta pesquera], aunque llegar allá era difícil" (Rivera et al. 2007: 92).

Un segundo punto a tratar dice relación con la frecuencia de partes esqueletarias registradas en la muestra y sus respectivas unidades de consumo. Para el caso de los vacunos, se aprecia una importante diversidad de partes, las que incluyen unidades que se asocian a cortes "oficiales" (osobucos y costillas) de mediano costo/beneficio (ver Tabla 1), relacionados muy probablemente con la preparación de cazuelas y guisos; unidades que son comercializadas tras su faenamiento (hueso sin carne), y adquiridas para la preparación de sopas de hueso; y sorprendentemente, elementos que tradicionalmente no se venden en el mercado, ya que son desechados en el lugar

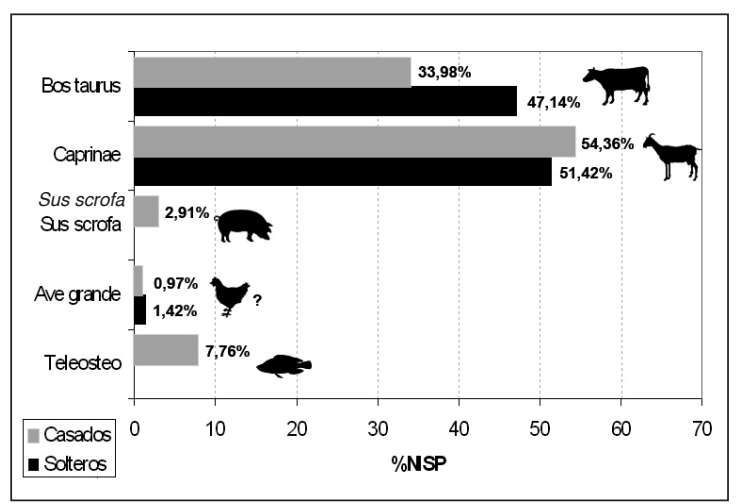

Figura 2. Frecuencia de taxones identificados en el área habitacional de Capote. de procesamiento. En esta última categoría se incluyen porciones de metapodios, falanges, cráneo y mandíbulas (Tabla 3). Contrariamente a lo esperado, la presencia de estas unidades sugeriría en principio, que los animales habrían sido faenados en el asentamiento, y que quizás éstos habrían llegado vivos a Capote. Resulta extraño, no obstante, que la carnicería no sea nombrada en los relatos etnográficos, por lo que nos inclinamos a pensar que fueron los comerciantes ambulantes quienes introdujeron en Capote unidades "no oficiales", de precio seguramente inferior. En cualquier caso, la presencia de estos segmentos con marcas de procesamiento, indica un aprovechamiento intensivo de la carcasa de bovino, lo que confirmaría su mayor escasez comparativa en relación con el caprino en el área.

Pese a la imposibilidad de detectar todos los cortes consumidos por los obreros de Capote, es posible agrupar las unidades óseas en los tres grandes conjuntos antes mencionados: huesos con carne (costillas y osobucos), huesos "pelados" y, huesos no listados oficialmente (metapodios, falanges, cráneo, mandíbula). Se aprecia en la Figura 3, que aunque tanto solteros como casados desechan fundamentalmente unidades utilizadas para hacer sopas y guisos, los casados tienen una mayor proporción de unidades con carne, las que tienen un mayor precio y obviamente, un mejor rendimiento. Volveremos sobre el tema del mayor número comparativo de restos de vacunos entre los solteros que en los casados. La explicación de esta situación, no necesariamente pasa porque los primeros "coman mejor", sino que éstos utilizan en sus preparaciones mayor

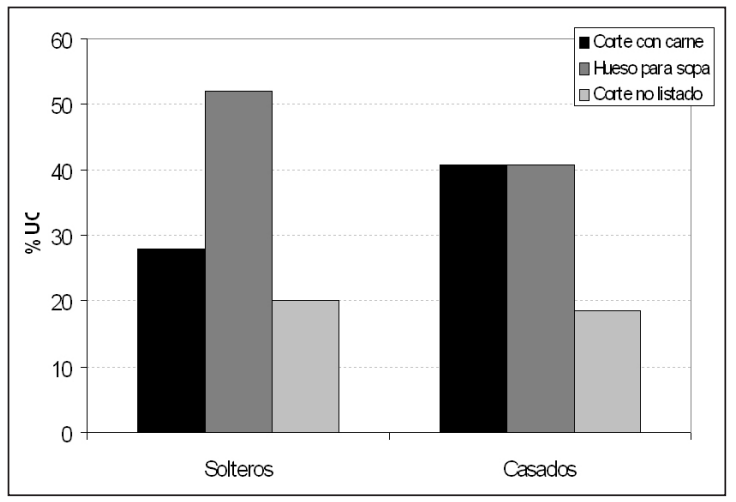

Figura 3. Porcentajes de unidades de consumo (\%UC) en el área habitacional de Capote (división según categorías analíticas explicadas en el texto). 
Rafael labarca, Elisa Calás, Francisco Rivera

\begin{tabular}{|c|c|c|c|c|c|c|c|c|}
\hline \multirow{3}{*}{ Parte esqueletaria } & \multicolumn{4}{|c|}{ Vacuno } & \multicolumn{4}{|c|}{ Caprino } \\
\hline & \multicolumn{2}{|c|}{ Solteros } & \multicolumn{2}{|c|}{ Casados } & \multicolumn{2}{|c|}{ Solteros } & \multicolumn{2}{|c|}{ Casados } \\
\hline & NISP & $\mathrm{UC}$ & NISP & $\mathrm{UC}$ & NISP & UC & NISP & $\mathrm{UC}$ \\
\hline Asta & - & - & 1 & - & - & - & - & - \\
\hline Cráneo & - & - & 5 & 1 & - & - & - & - \\
\hline Mandíbula & 3 & 2 & 1 & 1 & 1 & 1 & 4 & - \\
\hline Costilla & 5 & 5 & 8 & 8 & 7 & 5 & 16 & 13 \\
\hline Atlas & - & - & - & - & 2 & 2 & - & - \\
\hline Axis & - & - & - & - & 1 & 1 & - & - \\
\hline Vértebra cervical & 1 & 1 & 2 & 2 & 1 & 1 & - & - \\
\hline Vértebra torácica & 2 & 2 & 2 & 2 & 3 & 3 & 6 & 3 \\
\hline Vértebra lumbar & 3 & 2 & 2 & 2 & 3 & 2 & 1 & 1 \\
\hline Vértebra indeterminada & 1 & 1 & 3 & 3 & - & - & - & - \\
\hline Coxal & 1 & 1 & - & - & 2 & 1 & 3 & 3 \\
\hline Escápula & 2 & 2 & - & - & 1 & 1 & 3 & 3 \\
\hline Húmero px. & 1 & 1 & 1 & 1 & - & - & - & - \\
\hline Húmero df. & 1 & 1 & - & - & 2 & 2 & 2 & 2 \\
\hline Húmero ds. & 1 & 1 & 1 & 1 & 1 & 1 & - & - \\
\hline Radioulna px. & - & - & - & - & - & - & 3 & 1 \\
\hline Radioulna df. & - & - & - & - & 2 & 1 & 3 & 2 \\
\hline Radioulna ds. & - & - & - & - & - & - & - & - \\
\hline Metacarpo ds. & 1 & 1 & - & - & - & - & - & - \\
\hline Fémur px. & - & - & - & - & - & - & 1 & - \\
\hline Fémur df. & - & - & - & - & 3 & 3 & 4 & 3 \\
\hline Tibia df. & - & - & 1 & 1 & 4 & 3 & - & - \\
\hline Tibia ds. & 1 & 1 & - & - & - & - & - & - \\
\hline Astrágalo & - & - & 1 & 1 & - & - & - & - \\
\hline Calcáneo & - & - & 1 & 1 & - & - & - & - \\
\hline Metatarso px. & - & - & - & - & 1 & - & - & - \\
\hline Metapodio df. & - & - & - & - & 1 & - & 3 & - \\
\hline Metapodio ds. & - & - & 1 & 1 & 1 & - & - & - \\
\hline Falange i px. & - & - & 1 & 1 & - & - & - & - \\
\hline Falange i ss. & 2 & 2 & 1 & 1 & - & - & - & - \\
\hline Tarso/carpo & 2 & 2 & - & - & - & - & - & - \\
\hline Sesamoideo & 1 & - & 1 & - & - & - & - & - \\
\hline Molar & 1 & - & - & - & - & - & 4 & - \\
\hline Incisivo & 4 & - & 2 & - & - & - & 2 & - \\
\hline Total & 33 & 25 & 35 & 27 & 36 & 27 & 55 & 31 \\
\hline
\end{tabular}

Tabla 3. Resumen de partes esqueletarias de vacuno y caprino del área habitacional de Capote. 


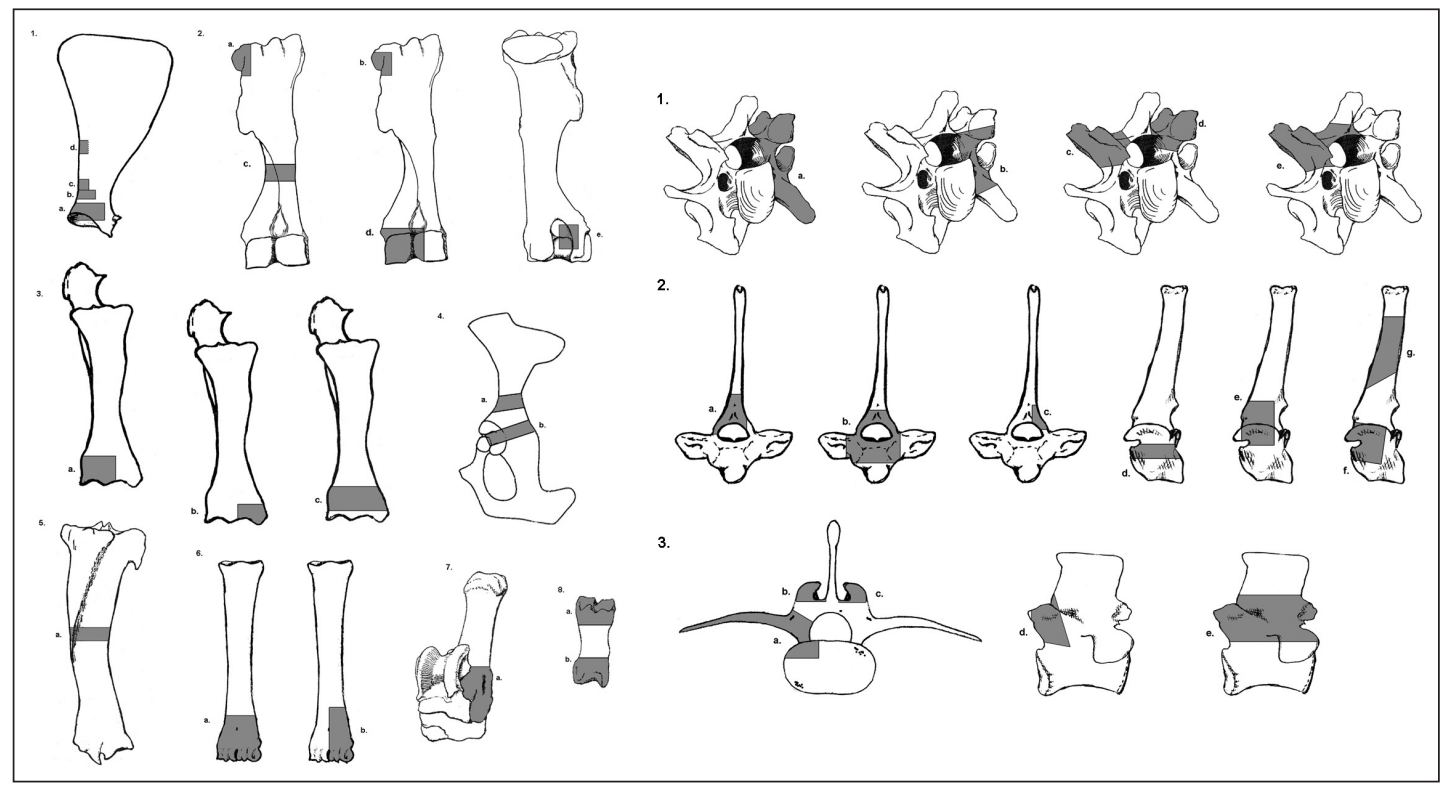

Figura 4. Unidades de Bos taurus con huellas de sierra en Capote.

número de unidades óseas para la elaboración de sopas, que tienen un costo inferior.

Por último, es importante mencionar la morfología de las unidades con huellas de sierra de vacuno. La principal característica que presentan es la impresionante diversidad de cortes, a tal punto que es imposible encontrar dos unidades similares (Figura 4). Por el momento no es posible explicar satisfactoriamente esta situación, considerando que sólo existen dos puntos de abastecimiento de carne y huesos en todo el asentamiento. Una explicación podría ser una reducción de piezas por parte de los propios consumidores con el objeto de maximizar los recursos, aunque no se puede descartar que se trate de distintos faenadores, particularmente teniendo en consideración que los vendedores ambulantes concurrían desde diversos sectores de la región (Rivera et al. 2007). Lo que sí es cierto es que esta alta variabilidad hizo imposible la detección de modos de procesamiento diferenciados (urbano vs. rural).

En cuanto a los caprinos, se registran diversas partes del esqueleto, encontrándose ausentes aquellas piezas de bajo rendimiento normalmente descartadas en los lugares de faenamiento (p.e., falanges, cráneo; ver Tabla 3). Esta situación confirma que los cabritos habrían sido un recurso más abundante y posiblemente de menor precio que el vacuno, lo que permitía a los capotinos consumir fundamentalmente aquellas unidades de más alto rendimiento cárneo. El análisis de las piezas óseas presentes y las huellas de sierra, indica que posibles unidades de faenamiento mayores (p.e., pierna) eran reducidas en porciones menores (unidades de consumo), con morfologías comparativamente más homogéneas que en el caso de los vacunos (Figura 5), relacionadas fundamentalmente con preparaciones secas ( $\sin$ sopas). Ante la ausencia de datos respecto de los precios de los cortes de cabrito, se han agrupado las unidades de consumo en función exclusivamente de su rendimiento cárneo, en tres grupos: alto, medio y bajo ${ }^{10}$ tomando como referente el índice de utilidad de oveja (Ovis aries) propuesto por Binford (1978). Se observa que, en general, tanto casados como solteros consumen unidades de alto rendimiento cárneo, las que suponemos eran las más apetecidas. Nuevamente se registran diferencias entre casados y solteros, esta vez más sutiles, las que indican una tendencia a un mayor consumo de unidades de más alto rendimiento entre los primeros (Figura 6).

\footnotetext{
${ }^{10}$ Se consideraron las siguientes unidades por categoría: Bajo: crá-
neo, mandíbula, atlas, axis, carpos, tarsos metapodios, astrágalo,
calcáneo, falanges; Medio: escápula, húmero px., húmero ds.,
radioulna px., radioulna ds., tibia px., tibia ds.; Alto: cervicales,

Se consideraron las siguientes unidades por categoría: Bajo: crá-
neo, mandíbula, atlas, axis, carpos, tarsos metapodios, astrágalo,
calcáneo, falanges; Medio: escápula, húmero px., húmero ds.,
radioulna px., radioulna ds., tibia px., tibia ds.; Alto: cervicales,

Se consideraron las siguientes unidades por categoría: Bajo: crá-
neo, mandíbula, atlas, axis, carpos, tarsos metapodios, astrágalo,
calcáneo, falanges; Medio: escápula, húmero px., húmero ds.,
radioulna px., radioulna ds., tibia px., tibia ds.; Alto: cervicales,

Se consideraron las siguientes unidades por categoría: Bajo: crá-
neo, mandíbula, atlas, axis, carpos, tarsos metapodios, astrágalo,
calcáneo, falanges; Medio: escápula, húmero px., húmero ds.,
radioulna px., radioulna ds., tibia px., tibia ds.; Alto: cervicales, torácica, lumbares, costillas, coxal, fémur px., fémur ds.
} 


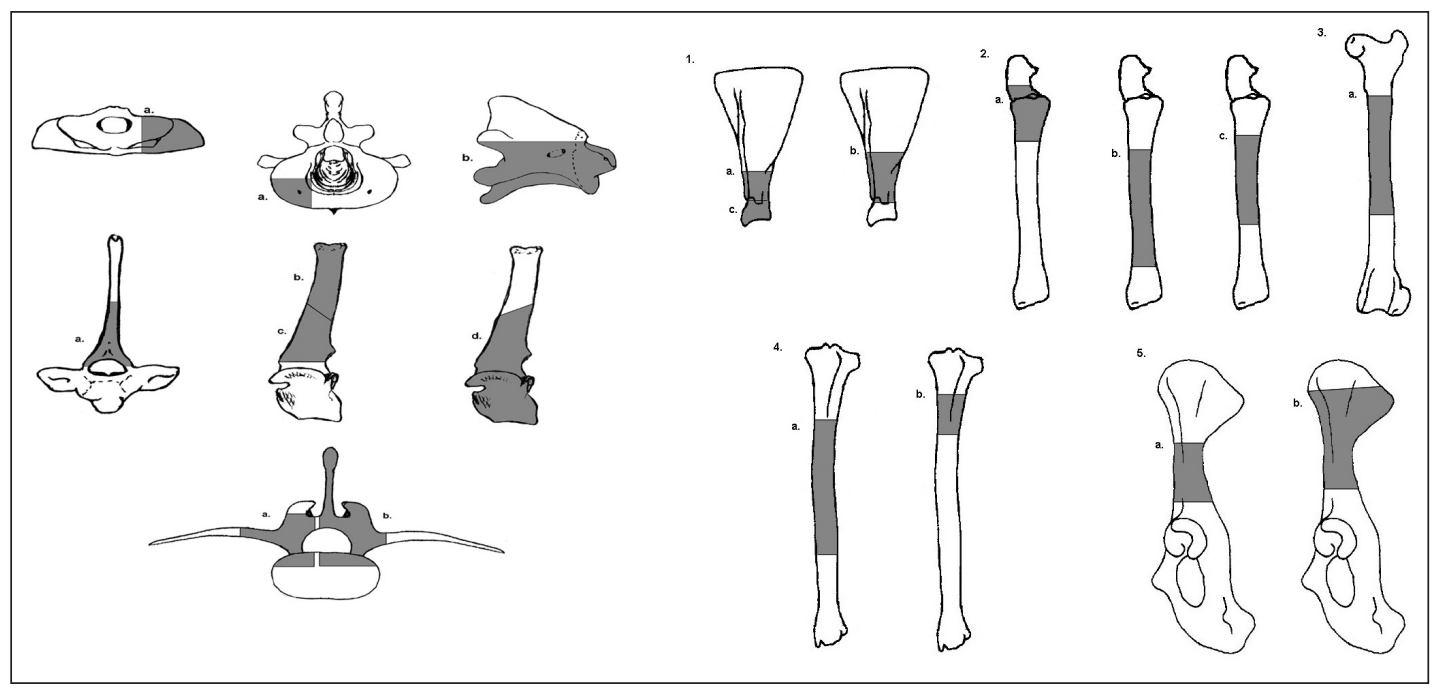

Figura 5. Unidades de Caprinae con huellas de sierra en Capote.

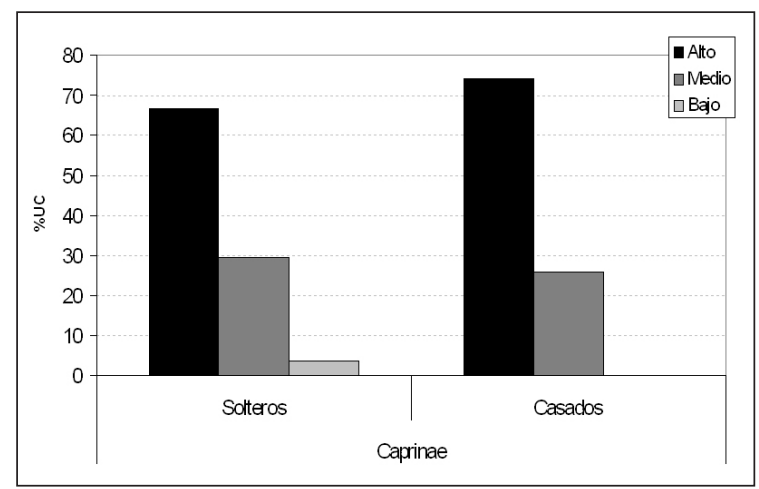

Figura 6. Rendimiento cárneo de las unidades de consumo (UC) de Caprinae en el área habitacional de Capote.

\section{$*$ Conclusiones}

El análisis faunístico centrado en la frecuencia de partes esqueletarias y en la diversidad de taxones presentes de dos sectores diferenciados del área habitacional de $\mathrm{Ca}$ pote, arrojó diferencias en las pautas alimenticias de los obreros solteros y casados (Tabla 4), confirmando en gran medida las expectativas enunciadas al comienzo.

Si bien esta investigación ha utilizado como marco de referencia los estudios de desigualdad social en zooarqueología histórica, las diferencias observadas entre solteros y casados no pueden ser atribuidas a distinciones de clases sociales, estatus socioeconómico o poder adquisitivo de estos dos grupos, ya que ambos poseen un salario similar y realizan labores similares al interior de la mina.

En este caso, son las decisiones femeninas ligadas con la economía doméstica las que se traducen en distinciones en el registro óseo. Así, las administradoras de las cantinas tendieron a comprar productos de inferior calidad, que en algunos casos se preparaban de manera más simple (como en sopas), ya que así disminuían los costos y se podía resolver de manera más sencilla la dura tarea que significaba 
Solteros o CASAdos: ¿QUiÉn COME MEJOR? LA EVIDENCIA FAUNística DEL ASENTAMIENTO MiNERO HiSTÓRICO DE...

\begin{tabular}{|l|l|}
\hline \multicolumn{1}{|c|}{ Casados } & \multicolumn{1}{c|}{ Solteros } \\
\hline Mayor diversidad de taxones & Menor diversidad de taxones \\
\hline Presencia de taxones exóticos (peces) & Ausencia de taxones exóticos \\
\hline $\begin{array}{l}\text { Mayor presencia de cortes asociados a carne en vacuno } \\
\text { (osobuco y costillas) }\end{array}$ & $\begin{array}{l}\text { Mayor presencia de cortes asociados a preparación } \\
\text { de sopas en vacuno }\end{array}$ \\
\hline $\begin{array}{l}\text { Menor presencia de unidades óseas no "tradicionales" en vacuno } \\
\text { (mandíbula, cráneo, metapodio, falanges) }\end{array}$ & $\begin{array}{l}\text { Mayor presencia de unidades óseas no "tradicionales" en } \\
\text { vacuno (mandíbula, cráneo, metapodio, falanges) }\end{array}$ \\
\hline Mayor número de unidades de alto rendimiento en caprinos & Menor número de unidades de alto rendimiento en caprinos \\
\hline
\end{tabular}

Tabla 4. Resumen de resultados obtenidos.

alimentar diariamente a los pensionistas solteros. Esta misma situación explica la ausencia de cerdo o pescado en los platillos de este último grupo, ya que implican técnicas distintas de preparación y una mayor inversión en tiempo y costos. En este sentido, un menú relativamente homogéneo facilita de mejor manera la labor culinaria.

En contrapartida, las dueñas de casa, al tener que alimentar a un número menor de individuos, con los que además poseen una relación afectiva, intentarán utilizar de mejor manera el presupuesto mensual, realizando variaciones y comprando productos de mejor calidad, en cantidades más reducidas.

Agradecimientos Esta investigación fue financiada por el proyecto FONDART 37081. Dos revisores anónimos colaboraron en mejorar la versión original del manuscrito.

\section{* Referencias citadas}

ADARO, L. y R. OLIVARES, 2006. Lecciones de anatomía veterinaria comparada (equino, bovino, cerdo). Departamento de Ciencias Biológicas Animales, Facultad de Ciencias Veterinarias y Pecuarias, Universidad de Chile, Santiago.

AMTMANN, G., 1967. Desposte de la carne de bovino en Santiago y su equivalencia anatómica. Revista de la Sociedad de Medicina Veterinaria $17(1-4): 55-70$.

BEHRENSMEYER, A. K., 1978. Taphonomic and ecologic information from bone weathering. Paleobiology 4 (2): 150-162.

1982. Time resolution in fluvial vertebrate assemblages. $P a-$ leobiology 8 (3): 211-227.

BEHRENSMEYER, A. K., K. D. GORDON y G. T. YANAGI, 1986. Trampling as a cause of bone surface damage and pseudotools. Nature 319: 768-771.

BINFORD, L., 1978. Nunamiut ethnoarchaeology. Academic Press, Nueva York.

1981. Bones: Ancient men and modern myths. Academic Press, Nueva York.
BOESSNECK, J., 1980. Diferencias osteológicas entre las ovejas (Ovis aries Linne) y cabras (Capra hircus Linne). En Ciencia y arqueología, D. Brothwell y E. Higg (Eds.), pp. 338-366. Fondo de Cultura Económica, México D. F.

BOESSNECK, J., H. MULLER y M. TEICHERT, 1964. Osteologische unterscheidungsmerkmale zwischen schaf (Ovis aries Linne) and ziege (Capra hircus Linne). Kuhn-Arch 78:1-129.

BOWEN, J., 1998. To market, to market: Animal husbandry in New England. Historical Archaeology 32 (3): 137-152.

CRABTREE, P., 1990. Zooarchaeology and complex societies: Some uses of faunal analysis for the study of trade, status, and ethnicity. En Archaeological method and theory, M. Schiffer (Ed.), pp. 155205. University of Arizona Press, Tucson.

CRADER, D. C., 1990. Slave diet at Monticello. American Antiquity 55: 690-717.

ELMERCURIO DE VALPARAÍSO n 34204, junio 17 de 1931.

GONZÁLEZ, S., 2002. Hombres y mujeres de la pampa. Tarapacá en el siglo de expansión del salitre. LOM Ediciones, Santiago. 
GRACIA, C., 1942. 888 recetas de cocina y repostería. Imprenta Puga Hnos., Santiago.

GRAYSON, D., 1984. Quantitative zooarchaeology. Academic Press, Orlando.

GUMERMAN, G., 1997. Food and complex societies. Journal of Archaeological Method and Theory 4 (2): 105-139.

HAYNES, G., 1983. A guide for differentiating mammalian carnivores taxa responsable for gnaw damage to herbivore limb bones. Paleobiology 9 (2): 164-172.

HUELSBECK, D. R., 1989. Zooarchaeological measures revisited. Historical Archaeology 23 (1): 113-117.

1991. Faunal remains and consumer behavior: What is being measured? Historical Archaeology 25: 62-76.

INSTITUTO NACIONAL DE NORMALIZACIÓN (INM), 1995. Cortes de carne de bovino. Norma chilena oficial. Santiago. 2003. Cortes de carne de ovino. Norma chilena oficial. Santiago.

JOHNSON, E., 1983. A framework for interpretation in bone technology. En Carnivores, human scavengers and predators: A question of bone technology, G. M. LeMoine y A. S. McEachern (Eds.), pp. 55-93. University of Calgary Archaeology Association, Calgary.

LA REFORMA DE CALAMA n 14, año 1, 1932.

LABARCA, R. y E. CALÁS, 2007 Ms. Informe arqueofaunístico de la mina histórica de Capote, III región.

LANDON, D. B., 1997. Interpreting urban food supply and distribution systems from faunal assemblages: An example from colonial Massachusetts. International Journal of Osteoarchaeology 7: 51-64.

2005. Zooarchaeology and historical archaeology: Progress and prospects. Journal of Archaeological Method and Theory 12 (1): 1-36.

LARRAÍN, L., 1943. Manual de cocina: Colección de recetas variadas y económicas. Imprenta San Francisco, Padre Las Casas.

LYMAN, R. L., 1977. Analysis of historic faunal remains. Historical Archaeology 11: 67-73.

1987. On zooarchaeological measures of socioeconomic position and cost-efficient meat purchases. Historical Archaeology 21 (1): 58-66.

1994. Vertebrate taphonomy. Cambridge Manuals in Archaeology. University Press, Cambridge.
MENGONI, L., 1988. El estudio de huellas en arqueofaunas: Una vía para reconstruir situaciones interactivas en contextos arqueológicos. Aspectos teórico-metodológicos y técnicas de análisis. En De procesos, contextos y otros huesos, N. Ratto y A. Haber (Eds.), pp. 17-28. Instituto de Ciencias Antropológicas, Buenos Aires.

1999. Cazadores de guanacos de la estepa patagónica. Colección Tesis Doctorales. Sociedad Argentina de Antropología, Buenos Aires.

MORVAN, H., 1938. Manual de cocina: Guía práctica de la dueña de casa. Editorial Zig-Zag, Santiago.

PEREIRA SALAS, E., 1977. Apuntes para la historia de la cocina chilena. Editorial Universitaria, Santiago.

PIZARRO, I., P. CAMPOS, C. MONTERO y R. CAMPUSANO, 2006. El valle de los naturales. Una mirada diaguita histórica al pueblo diaguita huascoaltino. Mosquito Comunicaciones, Santiago.

PRUMMEL, W. y H. J. FRISCH, 1986. A guide for the distinction of species, sex and body side in bones of sheep and goat. Journal of Archaeolgical Science 13:567-577.

REITZ, E., 1986a. Urban/rural contrasts in vertebrate fauna from the Southern Atlantic coast. Historical Archaeology 20 (2):47-58. 1986b. Vertebrate fauna from Locus 39, Puerto Real, Haiti. Journal of Field Archaeology 13:317-328.

RIVERA, F., 2008. Arqueología histórica en Capote: Organización espacial y diferenciación social en una mina de oro (s. XX). Memoria para optar al título de Arqueólogo. Departamento de Antropología, Facultad de Ciencias Sociales, Universidad de Chile, Santiago.

RIVERA, F., R. TAGLE, R. LORCA y D. PASCUAL (Eds.), 2007. Memorias de Capote. Patrimonio arqueológico-histórico de una mina de tres siglos. Consejo Nacional de la Cultura y las Artes, Santiago.

SCHMITT, D. N. y C. D. ZEIER, 1993. Not by bones alone: Exploring household composition and socioeconomic status in an isolated historic mining community. Historical Archaeology 27 (4): 20-38.

SCHULZ, P. D.y S. M. GUST, 1983. Faunal remains and social status. Historical Archaeology 17 (1): 45-53.

SHIPMAN, P., 1981. Applications of scanning electron microscopy to taphonomic problems. Annals of the New York Academy of Science 376:357-385. 